\title{
Long Term Follow Up of Bladder Amyloidosis
}

\author{
Daniel Ensley MD*, David Myers MD and George Kallingal MD \\ Brooke Army Medical Center, Fort Sam Houston, TX 78234, USA.
}

\begin{abstract}
This is an evaluation of a patient presenting with bladder amyloidosis and a seven year asymptomatic history between gross hematuria episodes. Cystoscopic evaluation showed a diffuse progression of amyloid deposits throughout the bladder. Biopsy confirmed the diagnosis and was negative for malignancy. Further evaluation ruled out systemic disease. To date, he has declined treatment. There is little available literature on long term urologic follow up for these patients. Bladder amyloidosis is rare, but most urologists will encounter it in their clinical practice. There are no agreed upon guidelines or recommendations for long term management of these patients, likely owing to its rarity. This patient had local progression confirmed on biopsy without evidence of malignancy. Evaluation for systemic amyloidosis was also negative. While a single, isolated case, it is unique for the length of follow up, the local progression and lack of symptoms. It is consistent with the low likelihood of systemic progression but does raise the question of how to treat these patients long-term. The authors recommend initial biopsy both to confirm diagnosis and rule out malignancy, evaluation for systemic disease and regular cystoscopic examinations to help direct therapy.
\end{abstract}

KEYWORDS

Amyloidosis, Bladder amyloidosis, Urology.

Correspondin\& Author Information

Dr. Daniel Ensley

Brooke Army Medical Center, Fort Sam Houston, TX 78234, USA. Tel: 210-539-0000.

Received: March 25, 2020; Accepted: April 14, 2020; Published: April 20, 2020

Copyright: (C) 2020 ASRJS. This is an openaccess article distributed under the terms of the Creative Commons Attribution 4.0 International license.

Citation: Daniel Ensley, David Myers, George Kallingal. Long Term Follow Up of Bladder Amyloidosis. Med Clin Res Open Access, 2020;1(1):1-3.

\section{Introduction}

Amyloidosis is a disease of unknown etiology in which proteins are deposited and if systemic can be progressively fatal. It was described in 1854 by Virchow and is characterized by betapleated sheets of aggregated amyloid protein (1). Up to one third of these patients will be diagnosed with multiple myeloma, B-cell lymphoma or other plasma cell neoplasia. It is composed of monoclonal light chains and is thought to be caused by a chronic inflammatory process. GU specific amyloidosis tends to be localized, with most cases occurring within the bladder. Amyloidosis can also be a systemic process which is ultimately fatal, however localized genitourinary disease does not appear to develop into systemic disease. Most cases have multiple sites of deposition with few cases showing diffuse deposition (2). The mean age of diagnosis is 55 years. Amyloidosis is occasionally encountered in Urology, usually during cystoscopic examination and is often mistaken initially for malignancy. While some cases of malignancy associated with amyloidosis have been documented, they are the exception not the rule. To date, there is no consensus regarding long term urologic care and there is a lack of guidance for long term disease management. Because of this, long term care can lead to repeat imaging studies or biopsies exposing the patient to unnecessary radiation or multiple rounds of anesthesia.

\section{Case Presentation}

The patient in question is a 71-year-old male who was referred to 
the Urology clinic for evaluation of a single, painless episode of gross hematuria. He denied any recent illness, voiding symptoms or trauma. His medical history is notable for hyperlipidemia and hypothyroidism. He has never used tobacco products and denied any chemical or environmental exposures. His family history was unremarkable for genitourinary diseases. He was incidentally discovered to have been seen by a Urologist in 2011 for a very similar episode. Cystoscopic biopsy at that time revealed small depositions in the bladder consisting of amorphous eosinophilic material throughout the tissue and surrounding vessels consistent with amyloidosis (Figure 1). He was lost to follow up before returning after this recurrent episode. The $\mathrm{CT}$ urogram showed a diffusely thickened bladder wall, unchanged from imaging done in 2011 and otherwise did not show any upper tract abnormalities. Cystoscopy revealed diffuse submucosal, heaped-up, yellow lesions with no areas of active hemorrhage (Figure 2).

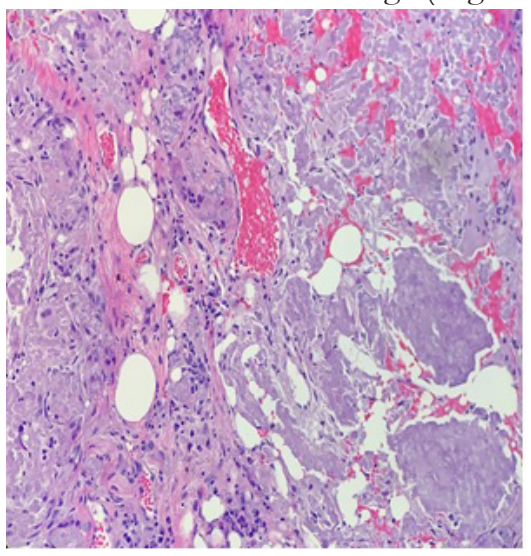

Figure 1: Patient biopsy from 2011 at original presentation, demonstrating diffuse amyloid deposition with reactive inflammation and changes within the bladder submucosa (H\&E).

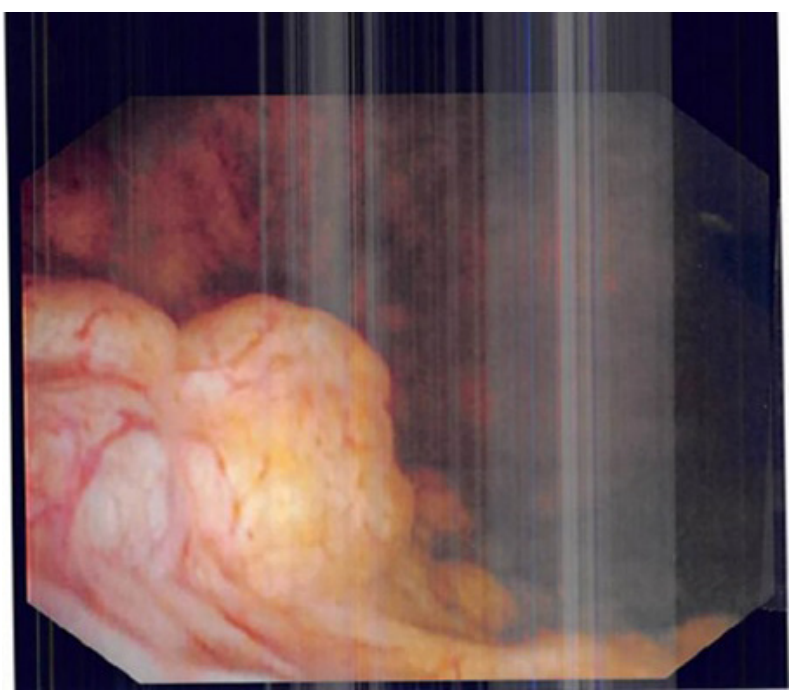

Figure 2: Cystoscopy from 2018 with heaped up lesions throughout bladder.

There were 3 calcifications adherent to the mucosa on the posterior and right anterior bladder walls (Figure 3). Labs were obtained. Urinalysis was normal, urine culture was negative for growth, PSA was within normal limits for his age group, complete Vol 1 Iss 1 metabolic panel and blood count were normal and finally, urine cytology was negative for evidence of high-grade urothelial carcinoma. Because the prior amyloid history was not yet known, cystoscopy under anesthesia with biopsy was performed and histopathologic analysis demonstrated amyloid deposition with associated reactive epithelial changes; carcinoma was not identified (Figure 4). A discussion was had with the patient and his primary care physician with regard to these findings and the need for further evaluation and follow up cystoscopic surveillance.

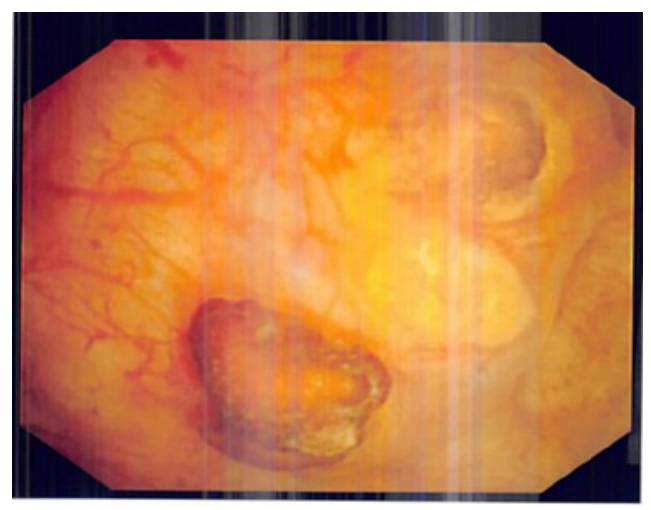

Figure 3: 2018 cystoscopy with Bladder wall calcifications.

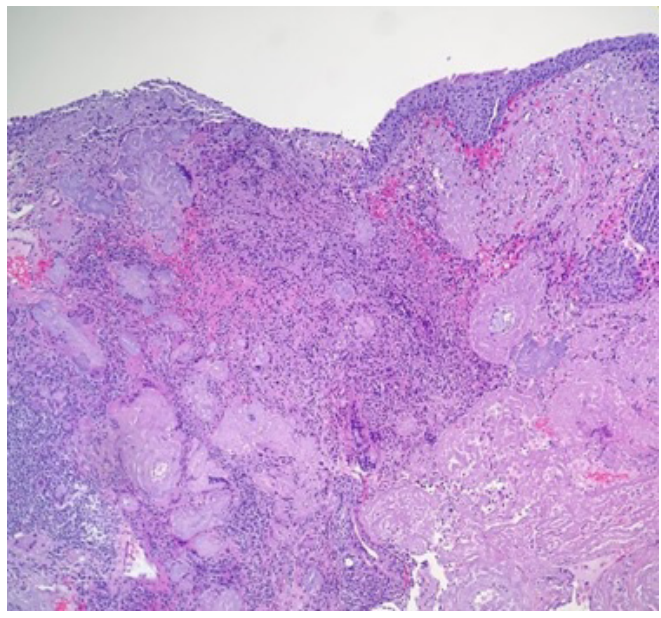

Figure 4: 2018 biopsy showing diffuse amyloid deposition.

\section{Discussion}

Gross hematuria is the most common presentation of localized amyloidosis of the bladder, occurring in over $75 \%$ of cases. Many patients also have LUTS or some degree of voiding dysfunction (1). Diagnosis is made through biopsy and histopathologic staining. Amyloid has an affinity for Congo Red special stain and shows apple-green birefringence under polarizing light microscopy (Figure 5).

The bladder is the most common site for urinary tract deposition and has a propensity to progress locally. (Mahmood, S. 2015) On cystoscopy, multiple deposits are most commonly seen while diffuse deposition occurs in only $\sim 10 \%$ of cases. Posterior and posterolateral walls were the most frequent site of deposition (4). The unique appearance is frequently confused for malignancy on initial cystoscopy, however no association has been made between Pages 2 of 3 
UCC and bladder amyloidosis. There are, however a few isolated cases of cancer in the literature (4). One patient had bladder cancer after 26 years of surveillance and six prior negative biopsies, highlighting the need for surveillance as well as underscoring the unique difficulties this disease presents (1). Urine cytology is not a high yield modality for surveillance or diagnosis as the amyloid deposits are submucosal and are rarely shed into the urine (5).

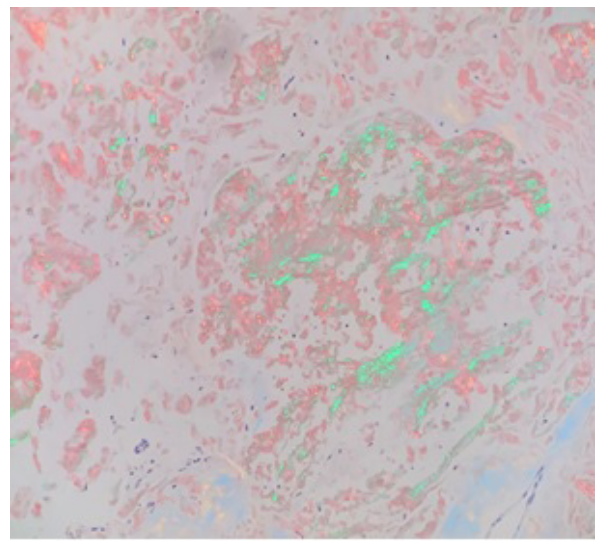

Figure 5: 2018 biopsy with Congo red special stain showing applegreen birefringence.

Standard treatment has been TUR for small localized deposits while more diffuse or recurrent treatment has been treated with multiple options including intravesical dimethylsulfoxide, oral colchicine, local radiation and partial or radical cystectomy $(3,6)$. Recurrence is common, occurring in up to $50 \%$ of cases, necessitating surveillance. However, there is no standardized follow up regimen owing to its rarity. A literature review places the number of documented primary and localized urologic amyloidosis at around 160-200 cases, mostly in single cases reports. Some authors have recommended 6-12 month surveillance cystoscopies as well as evaluation for systemic amyloidosis.

To date, there has been no evidence of a patient with localized bladder amyloidosis also having systemic disease, although the latter's progressive and ultimately fatal nature warrants evaluation at least in the initial presentation of suspected bladder localized disease. Evaluation includes biopsy samples of either rectal mucosa, salivary glands or abdominal subcutaneous fat, transthoracic echocardiography, 24-hour proteinuria and abdominal ultrasound (7).

\section{Conclusion}

This disease is sufficiently rare and long term follow up of bladder specific disease is rarer, still. This gentleman presented after a seven year, asymptomatic interval between gross hematuria episodes and subsequent evaluation. This case is unique for the long interval between follow up evaluations and lack of subjective symptoms. He had forgotten the initial diagnosis due to this long asymptomatic period essentially making it new workup and diagnosis. Radiographic imaging was non-specific and unchanged despite the long time period.

Cystoscopic examination showed a local progression of the disease in the bladder despite the patient's lack of subjective complaints and stability of disease on CT imaging. Pathologic evaluation confirmed the localized amyloidosis and ruled out urothelial cancer. Once malignancy and systemic disease has been ruled out, it is the author's belief that clinical picture and patient desires should guide management. While only a single case, it supports prior studies showing the stability of this disease and its disinclination for progression to cancer.

A long discussion was had with the patient and his primary care physician with regard to these findings and the need for further evaluation of systemic amyloidosis. Provided the patient remains asymptomatic and hematuria does not recur, he will follow up with cystoscopy surveillance at 12 month intervals.

\section{References}

1. Gupta P. Primary amyloidosis with high grade transitional cell carcinoma of bladder: A rare case report. J Cancer Res Ther, 2012;8(2):297-299.

2. Kawashima A, Alleman WG, Takahashi N, et al. Imaging evaluation of amyloidosis of the urinary tract and retroperitoneum. Radiographics, 2011;31(6):1569-1582.

3. Cooper, Christopher T. External beam radiation therapy for amyloidosis of the urinary bladder. Practical Radiation Oncology, 2017;8(1):25-27.

4. Tirzaman O. Primary localized amyloidosis of the urinary bladder: A case series of 31 patients. Mayo Clinic Proceedings, 2000;75(12):1264-1268. Proceedings, 75(12):1264-1268.

5. Zhou F, Lee P, Zhou M, et al. Primary localized amyloidosis of the urinary tract frequently mimics neoplasia: a clinicopathologic analysis of 11 cases. Am J Clin Exp Urol, 2014;2(1):71-75.

6. Varghese S, Wong NC, Shayegan B. Images in urology: Localized primary amyloidosis of the urinary bladder. Can Urol Assoc J, 2018;12(1):E42-E44.

7. Monge M. Localized amyloidosis of the genitourinary tract: report of 5 new cases and review of the literature. Medicine (Baltimore), 2011;90(3):212-22. 